\title{
Gut microbiota in children with juvenile idiopathic arthritis: characteristics, biomarker identification, and usefulness in clinical prediction
}

Xubo Qian ${ }^{1 \dagger}$, Yong-Xin Liü ${ }^{2+}$, Xiaohong Ye ${ }^{3}$, Wenjie Zheng ${ }^{4}$, Shaoxia $\mathrm{LV}^{5}$, Miaojun Mo ${ }^{6}$, Jinjing Lin ${ }^{7}$, Wenqin Wang ${ }^{8}$, Weihan Wang $^{3}$, Xianning Zhang ${ }^{9^{*}}$ and Meiping Lu ${ }^{1 *}$ (D)

\begin{abstract}
Background: Recent studies have suggested that the gut microbiota is altered in children with juvenile idiopathic arthritis (JIA). However, age, sex, and body mass index (BMI) were not matched in the previous studies, and the results are inconsistent. We conducted an age-, sex-, and BMI-matched cross-sectional study to characterize the gut microbiota in children with $\mathrm{JIA}$, and evaluate its potential in clinical prediction.

Methods: A total of 40 patients with JIA and 42 healthy controls, ranging from 1 to 16 years, were enrolled in this study. Fecal samples were collected for 165 rDNA sequencing. The data were analyzed using QIIME software and R packages. Specifically, the random forest model was used to identify biomarkers, and the receiver operating characteristic curve and the decision curve analysis were used to evaluate model performance.

Results: A total of 39 fecal samples from patients with JIA, and 42 fecal samples from healthy controls were sequenced successfully. The Chao 1 and Shannon-Wiener index in the JIA group were significantly lower than those in the control group, and the Bray-Curtis dissimilarity also differed significantly between the two groups. The relative abundance of 4 genera, Anaerostipes, Dialister, Lachnospira, and Roseburia, decreased significantly in the JIA group compared to those in the control group. The 4 genera included microbes that produce short-chain fatty acids (SCFAs) and were negatively correlated with some rheumatic indices. Moreover, 12 genera were identified as potential biomarkers by using the nested cross-validation function of the random forest. A random forest model constructed using these genera was able to differentiate the patients with JIA from the healthy controls, and the area under the receiver operating characteristic curve was 0.7975 . The decision curve analysis indicated that the model had usefulness in clinical practice.

(Continued on next page)
\end{abstract}

\footnotetext{
*Correspondence: zhangxianning@zju.edu.cn; meipinglu@zju.edu.cn

${ }^{+}$Xubo Qian and Yong-Xin Liu contributed equally to this work.

${ }^{9}$ Department of Genetics, Institute of Genetics, Institute of Cell Biology,

Zhejiang University School of Medicine, Hangzhou, Zhejiang Province, China

'Department of Rheumatology Immunology and Allergy, Children's Hospital,

Zhejiang University School of Medicine, Hangzhou, Zhejiang Province, China

Full list of author information is available at the end of the article
}

(c) The Author(s). 2020 Open Access This article is licensed under a Creative Commons Attribution 4.0 International License, which permits use, sharing, adaptation, distribution and reproduction in any medium or format, as long as you give appropriate credit to the original author(s) and the source, provide a link to the Creative Commons licence, and indicate if changes were made. The images or other third party material in this article are included in the article's Creative Commons licence, unless indicated otherwise in a credit line to the material. If material is not included in the article's Creative Commons licence and your intended use is not permitted by statutory regulation or exceeds the permitted use, you will need to obtain permission directly from the copyright holder. To view a copy of this licence, visit http://creativecommons.org/licenses/by/4.0/ The Creative Commons Public Domain Dedication waiver (http://creativecommons.org/publicdomain/zero/1.0/) applies to the data made available in this article, unless otherwise stated in a credit line to the data. 
(Continued from previous page)

Conclusions: The gut microbiota in patients with JIA is altered and characterized by a decreased abundance of 4 SCFA-producing genera. The decreases in the 4 genera correlated with more serious clinical indices. Twelve genera could be used as biomarkers and predictors in clinical practice.

Trial registration: The study is registered online at the Chinese Clinical Trial Registry on 11 May 2018 (registration number: ChiCTR1800016110).

Keywords: Juvenile idiopathic arthritis, Microbiota, Short-chain fatty acids, Butyrate, Propionate, Biomarker, Machine learning, Random forest model, Decision curve analysis

\section{Background}

Juvenile idiopathic arthritis (JIA) is the most common rheumatic disease in children and one of the more common chronic illnesses of childhood [1]. JIA represents a heterogeneous group of disorders, all of which share the clinical manifestation of arthritis. The worldwide incidence of JIA ranges from 0.8-22.6/100,000 children per year, with the prevalence rate ranging from 7 to $401 /$ 100,000 children per year [1-3]. JIA is the most common cause of joint disability and vision loss, and leads to a decreased health-related quality of life, impaired social functioning, and increased medical expenses [4-7].

Although the etiology and pathogenesis of JIA are not completely understood, immunogenetic susceptibility and an external trigger are considered as the main risk factors contributing to JIA [8-10]. Studies on twins have shown that the concordance rates for JIA among monozygotic twins range from 25 to $40 \%$ [11], which suggests that non-coding factors, including epigenetics, female sex, and environmental factors, play an important role in the pathogenesis of JIA. Environmental factors, such as tobacco exposure, infectious agents, vitamin D deficiency, and the gut microbiota, not only trigger the development of rheumatic diseases, but are also involved in the transition from the preclinical to clinical stage [12-15]. Accordingly, environmental factors are pivotal in the development and progression of JIA.

Of the environmental factors, the gut microbiota has been implicated in the pathogenesis of JIA [16-20], rheumatoid arthritis [21], and metabolic diseases [22]. For example, the gut microbiota in these patients is altered [16-21], some of which are considerably correlated with clinical indices in patients with rheumatoid arthritis, such as anti-citrullinated protein antibody, rheumatoid factor, and C-reactive protein [21]. Furthermore, the random forest models constructed using the microbiota are able to differentiate rheumatic patients from healthy controls [20, 21]. Additionally, some microbe components are detected in synovial fluid [23] and liver tissue [24], which can trigger autoimmune responses $[23,24]$. Finally, the causal relationships among the gut microbiome, short-chain fatty acids (SCFAs), and metabolic diseases have been demonstrated [22]. However, age, sex, and body mass index (BMI), which are the confounding factors that impact the composition of the gut microbiota [25-27], were not matched in some studies, and the results are inconsistent [17-20]. Whether the SCFA-producing genera dominate the differences between patients with JIA and healthy controls has not been well defined [17-20]. Furthermore, the clinical usefulness of the random forest models remains unclear $[20,21]$.

In order to address these problems, at least in part, we conducted an age-, sex-, BMI-, and ethnicity-matched cross-sectional study in Han Chinese children. We characterized the gut microbiota in patients with JIA, identified biomarkers, constructed a random forest model as a disease classifier using these biomarkers, and evaluated its usefulness in clinical prediction.

\section{Results}

Clinical and laboratory characteristics of the participants In total, 40 children with JIA and 42 healthy children (HC) were enrolled into the JIA group and the control group, respectively. The median ages in the JIA group and the control group were 10.27 years and 9.95 years, respectively. There were no statistical differences in age, sex, and BMI between the two groups (Table 1). The disease subtypes, activity parameters, and other clinical indices are also shown in Table 1 and Additional file 2: Table S1. Five children with JIA did not have cytokine data (Additional file 2: Table S1).

\section{Gut microbiota diversities differed between the JIA and control groups}

A total of 40 JIA stool samples and $42 \mathrm{HC}$ stool samples were collected, and 16S rDNA sequencing was completed in June 2019. In total, 39 JIA and $42 \mathrm{HC}$ stool samples were successfully sequenced, and 7347 operational taxonomic units (OTUs) were obtained after the removal of singletons. After OTUs less than $0.001 \%$ were filtered, the remaining OTUs were classified into 11 phyla, 19 classes, 32 orders, 58 families, and 94 genera (Additional file 2: Table S2 and Table S3). The Chao 1 and the Shannon indices, two commonly used $\alpha$ diversity indices, differed significantly between the two 
Table 1 Demographic and clinical characteristics of the two groups

\begin{tabular}{|c|c|c|c|c|}
\hline Characteristics & JIA group $(n=40)$ & Control group $(n=42)$ & Statistic & $P$-value \\
\hline Age, median (IQR) & $10.27(3.09-11.56)$ & $9.95(3.20-11.60)$ & $W=827$ & 0.907 \\
\hline Female & 20 & 20 & $x^{2}=0$ & 1.000 \\
\hline BMI, median (IQR) & $16.23(15.12-18.30)$ & $16.60(15.80-18.10)$ & $W=759$ & 0.455 \\
\hline Disease duration, months, mean (SD) & $3.47(1.45)$ & & & \\
\hline \multicolumn{5}{|l|}{ Subtypes of JIA } \\
\hline Oligoarthritis, n (\%) & $17(42.50)$ & & & \\
\hline Polyarthritis, n (\%) & $9(22.50)$ & & & \\
\hline Enthesitis-related arthritis, n (\%) & $14(35.00)$ & & & \\
\hline \multicolumn{5}{|l|}{ Disease activity parameters } \\
\hline CJADAS10, median (IQR) & $9(7-13)$ & & & \\
\hline ESR, median (IQR) & $20.50(10.50-36.00)$ & & & \\
\hline CRP, median (IQR) & $3.00(0.50-10.01)$ & & & \\
\hline \multicolumn{5}{|l|}{ Autoantibody status } \\
\hline ANA, median $(\mathrm{QQR})^{\mathrm{a}}$ & $0.00(0.00-4.60)$ & & & \\
\hline ACPA positive, n (\%) & $3(7.50)$ & & & \\
\hline RF positive, n (\%) & $3(7.50)$ & & & \\
\hline \multicolumn{5}{|l|}{ Cytokines } \\
\hline IL-2, mean (SD), pg/ml & $2.64(1.07)$ & & & \\
\hline IL-4, median (IQR), pg/ml & $2.10(1.30-2.07)$ & & & \\
\hline IL-6, median (IQR), pg/ml & $6.80(2.85-16.70)$ & & & \\
\hline IL-10, median (IQR), pg/ml & $2.90(2.15-3.90)$ & & & \\
\hline TNF, median (IQR), pg/ml & $2.00(1.15-2.40)$ & & & \\
\hline IFN- $\gamma$, median (IQR), pg/ml & $3.50(1.65-5.20)$ & & & \\
\hline \multicolumn{5}{|l|}{ Cluster of differentiation } \\
\hline CD3, mean (SD), \% & $70.60(8.14)$ & & & \\
\hline CD4, mean (SD), \% & $34.89(6.94)$ & & & \\
\hline CD8, mean (SD), \% & $29.58(7.70)$ & & & \\
\hline CD19, median (IQR), \% & $15.47(11.04-17.64)$ & & & \\
\hline CD3-CD16 + CD56+, median (IQR), \% & $10.47(6.64-13.80)$ & & & \\
\hline CD4/CD8, median (IQR) & $1.14(0.88-1.61)$ & & & \\
\hline
\end{tabular}

ACPA Anti-citrullinated protein antibodies, ANA Antinuclear antibody, BMI Body mass index, CD Cluster of differentiation, CJADAS10 Juvenile arthritis disease activity score 10, CRP C-reactive protein, ESR Erythrocyte sedimentation rate, IFN Interferon, IL Interleukin, IQR Interquartile range, $R F$ Rheumatoid factor, $T N F$ Tumor necrosis factor

${ }^{\mathrm{a}}$ Log10 transformed

groups $(P=0.0026$ and 0.031 , Wilcoxon test; Fig. 1a, Additional file 2: Table S4); however, there was no significant difference in the Simpson index between the two groups $(P=0.248$, Wilcoxon test; Additional file 1: Figure S3, Additional file 2: Table S4). The JIA and control groups had 3 and 8 unique genera, respectively; the two groups shared 83 genera (Fig. 1b, Additional file 2: Table S5). The Bray-Curtis dissimilarity, a commonly used $\beta$-diversity index, differed between the two groups $\left(P=0.019, \mathrm{R}^{2}=0.021\right.$, permutational multivariate analysis of variance [PERMANOVA] with 1000 Monte Carlo simulations; Fig. 1c). The phylogenetic tree, which was built using OTUs greater than $0.3 \%$, showed that these OTUs belonged to the following five phyla: Firmicutes, Bacteroidetes, Actinobacteria, Proteobacteria, and Verrucomicrobia (Fig. 1d, Additional file 2: Table S6). The power of the study was 0.89 (Dirichlet-Multinomial Model with 1000 Monte Carlo simulations).

\section{Alterations of the gut microbiota in JIA patients and its associations with clinical indices}

At the phylum level, the most common phyla in the two groups were the Bacteroidetes, Firmicutes, Actinobacteria, and Proteobacteria (Fig. 2a). The Proteobacteria had higher abundance in JIA group (4.56\%) as compared to that in the control group (4.03\%), and the 

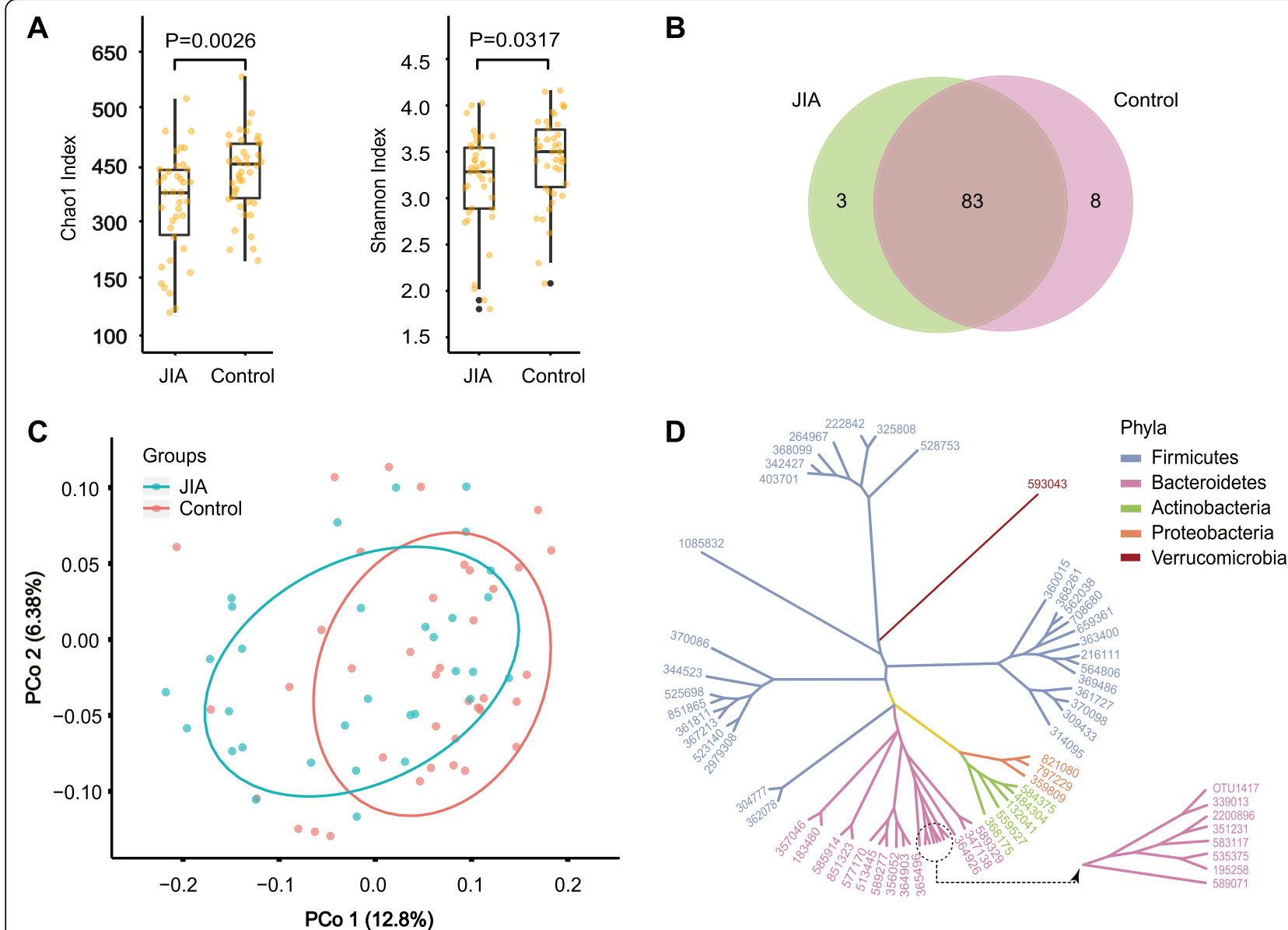

Fig. 1 Diversity analyses show that the differences in the $a$ - and $\beta$-diversities of the gut microbiota differ between the JIA and the control groups. a Comparisons of the Chao 1 and Shannon indices between the two groups. The two indices were significantly reduced in the JIA group compared to the control group ( $P=0.0026$ and 0.031 , Wilcoxon test). $\mathbf{b}$ Venn diagram based on genera. The two groups have 83 shared genera, with 3 unique genera in the JIA group and 8 unique genera in the control group. c Ordination plot for the first two PCoA axes based on BrayCurtis dissimilarity. The samples of the JIA and control groups are relatively clustered together, indicating that the Bray-Curtis dissimilarity differs between the two groups ( $P=0.019$, PERMANOVA test). $\mathbf{d}$ The phylogenetic tree was built using the OTUs greater than $0.3 \%$ (Additional file 2 : Table S6). The OTUs in the plot are colored by phyla

Verrucomicrobia was the opposite $(0.0036 \%$ vs $0.048 \%)$; which were significantly different when analyzed using ALDEx2 package $(P=0.033,0.029$, respectively; Wilcoxon test). However, none reached significance when the $P$-values were adjusted for multiple testing corrections using the Benjamini-Hochberg method.

The genera of Bacteroidetes, Bifidobacterium, Prevotella, Megamonas, and Lachnospira were dominant in the two groups (Fig. 2a, Additional file 2: Table S3). The 4 genera Anaerostipes, Dialister, Lachnospira, and Roseburia had lower abundance in the JIA group $(0.00$, $0.011,0.337$, and $0.66 \%$, respectively) as compared to those in the control group $(0.040,0.725,2.244$, and $1.162 \%$, respectively), which reached significance when the $P$-values were adjusted for multiple testing corrections using the Benjamini-Hochberg method (Adjusted
$P=0.031,0.013,0.041$, and 0.011 , respectively; Wilcoxon test; Additional file 2: Table S7). Of the 4 genera, Dialister was a genus of the Veillonellaceae family, the others belonged to the Lachnospiraceae family. All 4 genera belonged to the Clostridiales order and were found to be the microbes producing short-chain fatty acids (SCFAs) in previous studies [28-31]. No genera that were significantly enriched in the JIA group were identified by the Wilcoxon test (Adjusted $P>0.05$; Additional file 2: Table S7). The 4 genera were significantly correlated with 11 clinical indices (all adjusted $P$-values $<0.05$, "Holm" adjustment, Spearman's correlation; Fig. 2b). Among the 4 genera, the Lachnospira and Roseburia were correlated with 9 and 6 clinical indices, respectively, while the Anaerostipes and Dialister were only correlated with 2 and 1 clinical indices, respectively. Out of the 18 

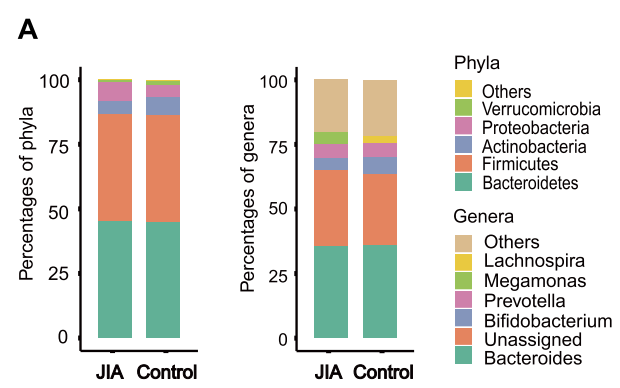

\section{C}

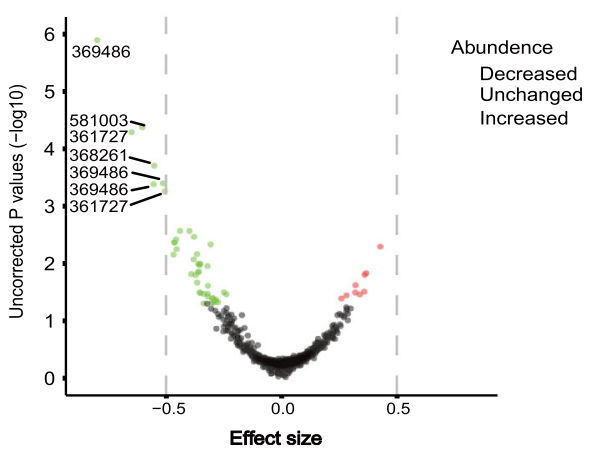

B

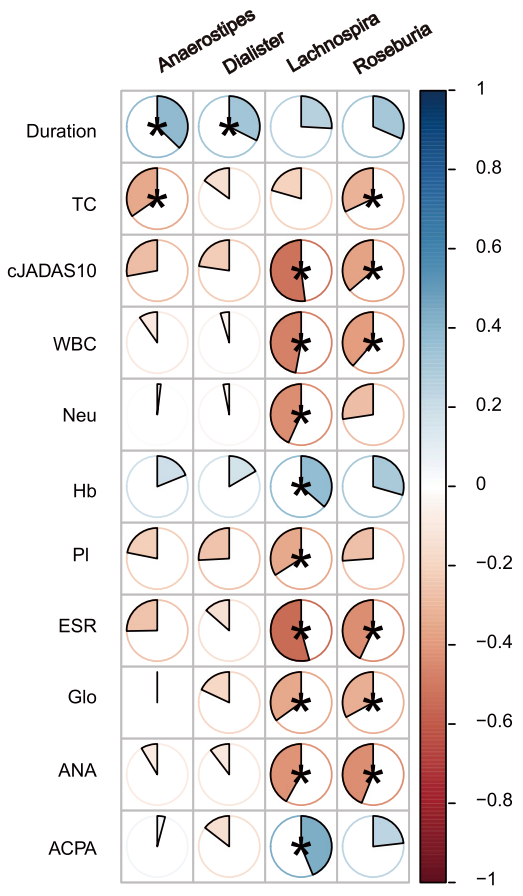

Fig. 2 The compositional differences at phylum, genus, and OTU levels, and associations between genera and clinical indices. a The compositional differences of the phyla and genera between the two groups. $\mathbf{b}$ Associations between the relative abundance of the 4 genera and clinical indices. A pie chart with an asterisk indicates that the correlation coefficient reached significance after the $P$-value was adjusted. c Volcano plot of the OTUs. Green and red points represent the sample of those with $P$-values $<0.05$ by Wilcoxon test (unadjusted $P$-values). The green and red colors indicate a decrease and increase in abundance, respectively. The effect size is the ratio of "the difference between groups" and "the maximum difference within groups." In general, the effect size cut-off is more robust than $P$-values. The OTUs are considered biological markers if their absolute values of effect size are $\geq 0.5$. Seven OTUs, marked with OTU numbers, have absolute values $>0.5$, including the five OTUs identified by Wilcoxon test (Additional file 2: Table S8). ACPA: Anti-citrullinated protein antibody; ANA: Antinuclear antibody; CJADAS10: Clinical juvenile arthritis disease activity score 10; Duration: Disease duration; ESR: Erythrocyte sedimentation rate; Glo: Globulin; Hb: Hemoglobin; Pl: Platelet; TC: Total cholesterol; WBC: While blood cell; Neu: Neutrophil

associations with adjusted $P$-values $<0.05,14$ were negatively correlated and 4 were the opposite (Fig. 2b). The absolute values of the correlation coefficients ranged from 0.319 to 0.544 (Fig. 2b). The first three associations with greatest correlation coefficients were the ones between Lachnospira and ESR, WBC, and ANA.

At the OTU level, 55 OTUs were significantly different in abundance between the two groups when analyzed by Wilcoxon test (Fig. 2c, Additional file 2: Table S8); however, only 5 OTUs, labeled as OTU_361727, OTU_368261, OTU_369429, OTU_581003, OTU_ 470382, and, differed between the two groups after the $P$-values were adjusted (corrected $P=0.002,0.015$, $0.017,0.037$, and 0.043 , respectively; the BenjaminiHochberg method; Additional file 2: Table S8). The 5 OTUs had lower abundance in the JIA group (0.004, $0.004,0.000,0.000$, and $0.010 \%$, respectively) than those in the controls $(0.158,0.093,0.132,0.015$, and $0.656 \%$, respectively). No OTUs with increased relative abundance were identified by the Wilcoxon test after the $P$ values were adjusted.

\section{Twelve genera have the potential to serve as biomarkers} in JIA diagnosis

To explore whether the gut microbiota can be used as biomarkers to differentiate JIA patients from healthy controls, we constructed six random forest models using all microbiota members at the phylum, class, order, family, genus, and OTU levels (Additional file 1: Figure S4). The model constructed using the microbiota at the genus level showed the best predictive accuracy of $67.9 \%$ (e.g., the out-of-bag error rate was 32.1\%) among all taxonomic levels (Additional file 1: Figure S4). The results of the ten-fold nested cross-validation showed that as the predictors (e.g., variable or genus numbers in this case) increased, the out-of-bag error rate decreased sharply. When the genus number exceeded 12, the error rates no longer decreased (Fig. 3a). It indicated that the optimal number of biomarkers (genera) was 12 . The 12 genera with highest variable importance are shown in Fig. 3b. Of the 12 genera, 10 had a lower abundance in the JIA group compared to the controls, while the other 2 genera (Faecalibacterium and Oscillospira) were the 

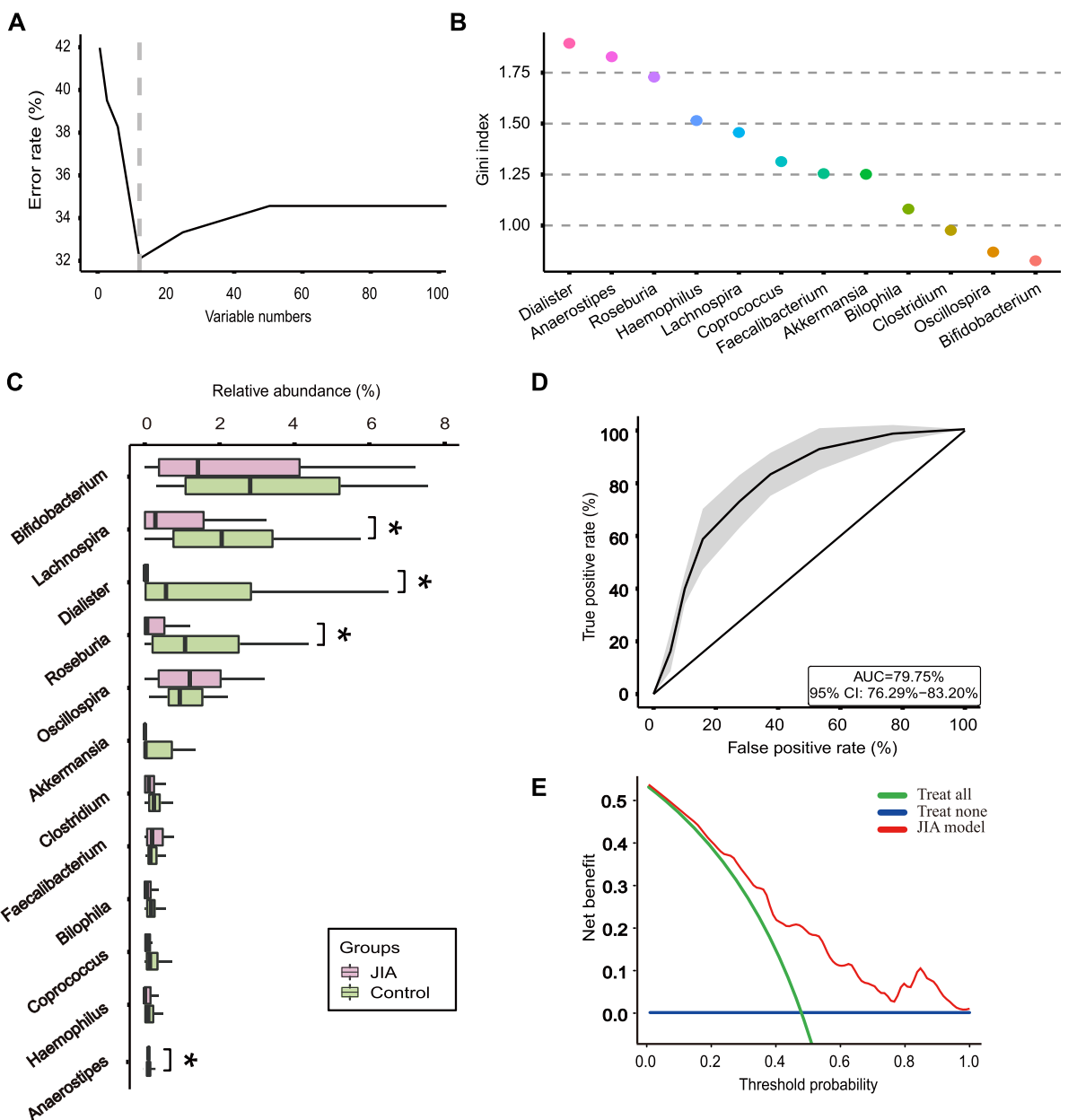

D

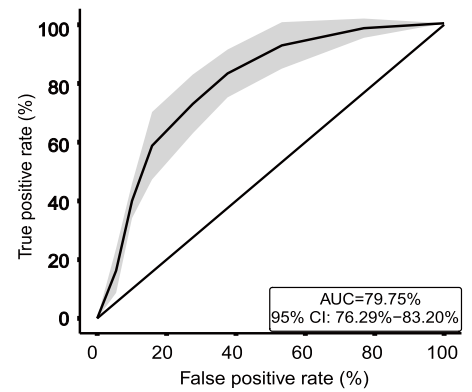

E

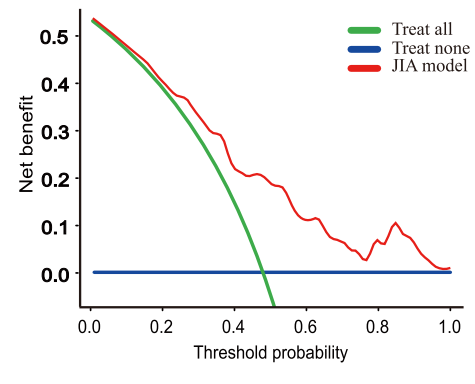

Fig. 3 The random forest model constructed using 12 genera can be used as a disease classifier to differentiate JIA patients from healthy controls. a Plot of genera numbers vs error rates. As the genera numbers increased, the error rates decreased sharply. The dashed gray line marks the optimal cut-off for biomarker selection. This analysis indicated that 12 was the optimal predictor (genus) number. b The variable importance of the genera analyzed using the randomForest package in $\mathrm{R}$. The most important 12 genera are listed in the plot. The greater the Gini indices, the more important the variables are. c The relative abundance of the 12 genera identified by the random forest model and Wilcoxon test. The 4 genera marked with an asterisk differed significantly in abundance between the two groups by Wilcoxon test (corrected $P<0.05$ ). $\mathbf{d}$ ROC of the random forest model constructed using the 12 genera. The diagonal line in the graph marks an AUC of 0.5. The 95\% confidence intervals are shown as shaded areas. e DCA for the random forest model constructed using the 12 genera. The $y$-axis measures the net benefit. The green line represents the situation with the assumption that all children received treatment due to JIA. The blue line indicates the net benefit under the assumption that no children received treatment due to JIA (e.g., representing the natural disease course without medical intervention so that the net benefit is constantly zero). The red line is above the green and blue lines, especially within the threshold probability of $0.23-0.77$, which implies that the prediction model is able to achieve a greater net benefit than the situation when the children are treated or untreated without any model

opposite (Fig. 3c). The 12 genera identified by the random forest method included the 4 genera that were identified by Wilcoxon test (Fig. 3c); at the OTU level, the two analysis methods identified similar results (Additional file 1: Figure S5).

After the 12 genera (biomarkers) were identified, we constructed a new random forest model as a disease classifier using these genera (Additional file 1: Figure S2). The out-of-bag error rate of the new model was $33.30 \%$, which was only slightly higher than that of the model constructed using all microbiota members at the genus level. The area under the receiver operating characteristic curve (AUC) was 0.7975 (Fig. 3d). We used another tool, known as decision curve analysis (DCA), to evaluate the new model; the results of which showed that the net benefit obtained by the model was greater than the "treat-all" and "treat-none" curves within the threshold probability between 0.23 and 0.77 (Fig. 3e).

\section{Discussion}

With the rapid development of sequencing technologies [32-35] and bioinformatics analysis methods [36, 37], 
the associations between microbiome and diseases have been demonstrated in recent years. The gut microbiota was found to be associated with rheumatic diseases approximately 50 years ago [38], and in the last 10 years, the involvement of the gut microbiota in the development and progression of JIA and other rheumatic diseases has been further established [24, 39]. In the current study, we found that the abundance of the 4 genera, Anaerostipes, Dialister, Lachnospira, and Roseburia, decreased in JIA patients, which were found to be the SCFA-producing microbes in previous studies [2831]. The decreases in the 4 genera were correlated with more serious clinical indices. Moreover, we constructed a random forest model as a disease classifier using 12 biomarkers (genera), which was demonstrated that it had usefulness in clinical prediction.

Age is one of the main factors impacting the composition of the gut microbiota [25,40], similar to sex [26], ethnicity [41], and others [42]. Thus, only age-, sex-, and ethnicity-matched healthy controls were selected in order to minimize confounding bias. Over the last decade, systemic JIA has increasingly been considered as an autoinflammatory condition, rather than an autoimmune disease. This distinguishes systemic JIA from other subtypes $[43,44]$; thus, children with systemic JIA were not included in the current study.

The diversity in a single ecosystem or sample, called $\alpha$-diversity, is often measured by the Chaol, ShannonWiener, and Simpson indices. The Chao1 and ShannonWiener indices give more weight to rare species, whereas the Simpson index puts more emphasis on common species [45]. In this study, the Chaol and Shannon-Wiener index differed considerably between the two groups, but the Simpson index did not, implying that the rare species contributed to the differences to a greater extent than the common species. The results of the $\alpha$-diversity analyses were similar to those of previous studies [17, 46]; however, other studies did not find any differences in the $\alpha$-diversity indices [18-21].

Bray-Curtis dissimilarity, a commonly used $\beta$-diversity index, differed between the two groups, which indicated that the two groups had a different composition of gut microbiota. However, the $\mathrm{R}^{2}$ value was 0.02132 , which showed that only $2.3 \%$ of the total variance could be explained by the group, e.g., JIA contributed to $2.3 \%$ alterations of the gut microbiota composition. The result of the $\beta$-diversity index was similar to those of previous studies [17, 20, 46, 47]. However, other studies either failed to find any differences in $\beta$-diversity indices or did not have relevant data $[18,19,21]$. The inconsistent $\alpha-$ and $\beta$-diversity results among these studies may be attributed to, at least in part, study design, study population, composition of JIA subtypes, duration of disease, and medication use prior to enrollment [39].
Diversity analyses have revealed that the two groups had a different composition of microbiota. Univariate community analysis further demonstrated the microbiota differences between the two groups, which were found to be the SCFA-producing microbes [28-31]. More specifically, at the genus level, the relative abundance of Anaerostipes, Dialister, Lachnospira, and Roseburia in JIA patients decreased significantly (corrected $P<0.05$, Wilcoxon test), three of which are butyrateproducing microbes including Anaerostipes [28, 29], Lachnospira [30], and Roseburia [31]. The other genus, Dialister, is a propionate-producing microbe [48]. Compared to previous studies, decreases in the abundance of the Anaerostipes or Lachnospira were also observed in patients with JIA [18, 47], but these results were opposite to those observed in patients with rheumatoid arthritis $[21,46]$. In contrast to the results in our study, the abundance of Dialister was increased in the previous study [49]. Hence, the findings of microbiota changes are inconsistent. Our study used the same sequencing method and similar data analysis methods as the previous studies. The key difference between them was the control selection. That is, whether the confounding factors of age, sex, BMI, and ethnicity were matched mainly contributed to the differences in the results between our study and others, because these confounding factors definitely impact the composition of gut microbiota [25-27, $40,41]$. Of these confounding factors, age probably is the main one, because the composition of gut microbiota in children younger than 16 years old varies substantially $[25,50,51]$. To some extent, other factors such as geography and diet may also have an effect on the differences $[40,52]$. It has been demonstrated that SCFAs, including acetate, propionate, butyrate, and pentanoate, have considerable immunomodulatory effects through several pathways, such as inducing the differentiation of regulatory $\mathrm{T}$ cells, enhancing IL-10 production, and suppressing Th17 cells [53-55]. Moreover, butyrate administration suppressed the expression of inflammatory cytokines and ameliorated collagen-induced arthritis in mice [54]. Although causal relationships among gut microbiota, SCFAs, and metabolic diseases have been demonstrated previously $[22,56]$, these relationships remain vague in JIA and need to be further investigated.

Similar to a previous study [21], the correlations between the gut microbiota and some clinical indices were verified in the current study, but the absolute values of the correlation coefficients were relatively small. The 4 genera, especially the Lachnospira and Roseburia, were mainly negatively correlated with the clinical indices, e.g., when the relative abundance of the 4 genera decreased, the rheumatic clinical indices became higher or more active. This implies that, as in the previous study regarding metabolic diseases [22], the following 
sequential changes may be observed: Microbiota alterations (decrease in the abundance of SCFA-producing microbes), low concentrations of SCFAs, immune dysfunctions, and eventually rheumatic diseases.

The diagnosis of JIA primarily depends on medical history, physical findings, and the exclusion of other diseases, and is a challenging task in clinical practice. The 12 genera identified in our study were used to construct a new random forest model, which could help physicians to establish a diagnosis of JIA. The performance of a prediction model is usually evaluated with ROC, which assesses how well the predicted risks distinguish between patients with and without disease. Nevertheless, ROC cannot be used to evaluate whether a model could improve clinical decision making $[57,58]$. In 2006, the DCA was introduced to overcome this limitation [59]. In our study, the AUC of the new model was $79.75 \%$, which was lower than those reported in some earlier studies $[20,21]$, while the other studies did not report ROC results $[17-19,23,46,47]$. The DCA analysis showed that our prediction model had greater clinical usefulness than the situation when the children were managed without a prediction model, particularly for those within the threshold probability of $0.23-0.77$ [60]. However, the curve between the threshold probability of $0-0.23$ is close to the green line (Fig. 3e), indicating that the additional gain of the model was not significantly different from the "treat-all" model where the threshold probability ranged from 0 to 0.23 [58]. To the best of our knowledge, our study is the first one to investigate the clinical usefulness of a random forest model based on the gut microbiota by using DCA. It remains unclear whether the prediction model can be further improved by integrating the clinical characteristics. Further studies of the model based on the gut microbiota are warranted to refine its application in clinical decision making.

There were several limitations to our study. First, oligoarthritis, polyarthritis, and enthesitis-related arthritis were lumped together in the JIA group. Second, the proportion of enthesitis-related arthritis among the subtypes was relatively high; thus, whether the results can be extrapolated to other study populations requires to be determined. Third, the sample size was relatively small due to the rigorous inclusion criteria; moreover, we did not conduct a longitudinal study. Lastly, we did not detect the SCFAs in fecal, blood, and synovial samples; thus, whether the decreased abundance of SCFA-producing genera leads to the concentration changes in these samples remains to be confirmed.

\section{Conclusions}

In summary, this study shows that the gut microbiota is altered in patients with JIA, and is characterized by a decreased abundance of SCFA-producing genera, including
Anaerostipes, Dialister, Lachnospira, and Roseburia. The 4 genera, especially the Lachnospira and Roseburia, were mainly negatively correlated with the clinical indices, e.g., when the relative abundance of the 4 genera decreased, the clinical indices became higher or more active. Furthermore, the random forest model constructed using 12 genera could accurately predict individuals with or without JIA, which indicates the 12 genera could be used as biomarkers and predictors in clinical practice. Further studies are warranted to explore the causal relationships among gut microbiota, SCFAs, and JIA, and to refine the model's application in clinical decision making.

\section{Methods}

\section{Study design, participants, and settings}

We performed an age-, sex-, BMI-, and ethnicitymatched cross-sectional study at two tertiary hospitals between June 2018 and May 2019. All children were enrolled at the Children's Hospital, Zhejiang University School of Medicine, and the Jinhua Municipal People's Hospital, which are tertiary hospitals in Zhejiang Province, located in the southeastern part of China. The study had two groups: study group (JIA group) and control group (healthy controls). The flowchart of the study is shown in Additional file 1: Figure S1. The healthy children were those who visited their physicians for routine physical examinations at the two hospitals. The children with JIA met the following inclusion criteria: 1) Aged between 1 and 16 years old; and 2) new-onset JIA diagnosed according to the International League of Associations for Rheumatology classification criteria [61]. Specifically, new-onset was defined as disease duration between 6 weeks and 6 months, and absence of any treatment with disease-modifying anti-rheumatic drugs (DMARDs), biologic therapy, or steroids (ever). Healthy controls were age-, sex-, BMI-, and ethnicity-matched individuals with no history of JIA or the diseases listed in the exclusion criteria.

The exclusion criteria applied to both groups were as follows: 1) Children who were unwilling to participate in the study; 2) patients with systemic JIA; 3) individuals who had malnutrition [62] or were overweight [63]; 4) patients with recent $(<3$ months prior) use of any antibiotics or probiotics; and 5) patients with co-morbidities including inflammatory bowel disease, allergic disorders, diabetes, primary immunodeficiency, tumors, or other chronic diseases.

\section{Sample collection and measurement methods}

Specimens used in this study included fecal and blood samples. The former were used for $16 \mathrm{~S}$ rDNA sequencing, and the latter were used to determine clinical indices such as complete blood cell count, biochemical 
profile, and serum cytokines. Fecal and blood samples from the JIA patients were collected within $24 \mathrm{~h}$ after the patients were admitted to the two hospitals, and fecal samples from the healthy controls were collected mainly at home. The fecal samples were stored at $20^{\circ} \mathrm{C}$ within $15 \mathrm{~min}$ after collection, and then were transferred to our laboratory on dry ice within $24 \mathrm{~h}$ of collection and stored at $-80^{\circ} \mathrm{C}$ thereafter. Microbial DNA was extracted from the fecal samples using the TIANamp Stool DNA Kit (TIANGEN Biotech [Beijing] Co., Ltd., Beijing, China). A polymerase chain reaction was performed using 10-100 ng microbial DNA and $10 \mu \mathrm{M} \mathrm{V} 3-\mathrm{V} 4$ primers targeting 341F (CCTACGGGNG GCWGCAG) and 805R (GACTACHVGGGTATCTAATCC) and Phanta Max Master Mix (Vazyme Biotech Co., Ltd., Nanjing, China). Samples were pooled to equal concentrations, then sequenced on one lane of a MiSeq platform using the MiSeq Reagent Kit v3 (600 cycles; Illumina Inc., Shanghai, China), generating at least 30000 reads per sample.

Complete blood cell count was determined using a hematology analyzer (Mindray BC5310, Mindray Corp., China). Biochemical profile, such as alanine aminotransferase, aspartate aminotransferase, and creatinine, were determined using an automatic biochemical analyzer (HITACHI 7600, Hitachi Ltd., Japan). Serum cytokines including IL-2, IL-4, IL-6, IL-10, TNF- $\alpha$, and IFN- $\gamma$ were determined by the CBA Human Th1/Th2 Cytokine Kit II (BD Biosciences, USA) using a cell analyzer (BD FACSCanto $^{\mathrm{mat}}$ II, Amersham Biosciences Corp., USA). Immunoglobulins were determined using a photometric assay analyzer $\left(\operatorname{cobas}^{\circ}\right.$ c 702 module, Roche Corp., Swiss). Autoantibodies, such as anti-citrullinated protein antibodies and antinuclear antibody, were determined using an immunofluorescence quantitative analyzer (HELIOS IOS-1000-AES, AESKU, Germany) and an automated western blot processor (Blotray-866, Rayto Life and Analytical Sciences Co., Ltd., China).

\section{Bioinformatics analysis}

The QIIME pipeline [64] was used to process the sequencing data. All of the downstream analyses including diversity analyses and visualizations, differential abundance testing, correlation analysis, and biomarker identification and its evaluation were performed in $\mathrm{R}$ 3.6.1 (https://cran.r-project.org/).

\section{The processing of $16 \mathrm{~S}$ rRNA sequencing data}

The detailed methods are available in the literature [65]. We ran open-reference OTU picking at $97 \%$ identity, and then feature sequences were assigned to taxonomic classification using the classifier based on the Greengenes 13.5 database [66]. Samples with less than 1000 sequences per sample were considered failures and filtered out, and OTUs less than $0.001 \%$ were removed $[42,67]$.

\section{a-Diversity measures and calculations}

$\alpha$-diversity indices, such as the Chao 1, ShannonWiener, Simpson, and Pielou's evenness indices, were calculated using the vegan package $2.5-5$ [68].

\section{$\beta$-Diversity measures and comparisons}

The Bray-Curtis dissimilarity, a commonly used $\beta$ diversity index, was used in our study, and was calculated using the distance() function (a function of $\mathrm{R}$ package was expressed as "function name()") in the phyloseq package 1.26.1 [69]. The Bray-Curtis dissimilarity between the two groups was compared by the adonis() function in the vegan package [68]. In order to plot the figure of principal coordinate analysis $(\mathrm{PCoA})$, three steps were carried out: 1) The zero values in the OTU table were replaced using the $\mathrm{zCompositions}$ package 1.3.2-1 [70]; 2) the data were transformed using the decostand() function (Hellinger transformation, from the vegan package) in order to alleviate the horseshoe effect [71]; 3) PCoA was performed using cmdscale() in the vegan package [68].

\section{Visualization of the phylogenetic tree}

The phylogenetic tree was plotted using the ggtree package 1.14.6 [72].

\section{Differential abundance testing}

The differential abundance testing was performed using the ALDEx2 package 1.16.0 [73]. The effect size and $P$ values, generated by this analysis, were used to draw a volcano plot, which was visualized with the ggplot2 package 3.2.1 [74].

\section{Correlation analysis of the genera and the clinical indices}

Spearman correlations between the genera and clinical indices were calculated using the psych package 1.8.12 [75], and visualized using the corrplot package 0.84 [76].

\section{Identification of the genera serving as biomarkers}

The built-in $\mathrm{rfcv}()$ function from the randomForest package 4.6-14 was used to explore the relationship between genera number and error rate [77, 78]. A nested cross-validation procedure was implemented in order to select an optimal predictor number using the $\operatorname{rfcv}()$. The number corresponding to the minimum error rate was considered as an optimal predictor number or biomarker number. The OTU table at the genus level, which contained the selected biomarkers (biomarker data), was used for downstream analyses. The flowchart of the major steps involved in biomarker identification is shown in "Additional file 1: Figure S2". 


\section{Construction of the receiver operating characteristic curve (ROC)}

Ten-fold cross-prediction based on the biomarker data was performed to construct the ROC, i.e., the input samples (the biomarker data) were partitioned into 10 subsets, 9 subsets were used to fit random forest models in the randomForest package [78], and the rest subset was used to calculate prediction probability in the ROCR 1.0-7 [79]. This cross-prediction process was then repeated 10 times, with each of the 10 subsets used exactly once as the prediction data. The 10 sets of prediction results were used to calculate $95 \%$ confidence intervals and plot the ROC.

\section{Decision curve analysis (DCA)}

Two main steps were performed to plot the DCA curve: Step 1: a random forest model based on the biomarker data was fitted in the randomForest package [80], and step 2: votes, a probability matrix produced in random forest process, and sample metadata file were used to plot the DCA curve in the $\mathrm{R}$ function that is detailed in the reference [60]. The net benefit and threshold probability were calculated as previously described [57].

\section{Sample size and power calculations}

No formal sample size calculation was performed before the participants were enrolled.

The sample size was bigger than those used in previous studies $[18,19]$. After the study was completed, the power of the study was calculated using the DirichletMultinomial Model in the R package HMP [81].

\section{Statistical analysis}

Continuous data were expressed as mean (standard deviation) and tested by Student's $t$ test or median with interquartile ranges and analyzed by Wilcoxon test. Categorical data were presented as percentages and were tested by Chi-square analysis or Fisher's exact test. Spearman's rank correlation was used to analyze correlations between non-normal data. A few children with JIA did not have cytokine data. These cytokine data were treated as missing values when performing correlation analysis in the $\mathrm{R}$ package psych [75]. All tests were twosided tests, and $P<0.05$ was considered statistically significant. $P$-values were adjusted for multiple testing using the "Benjamini-Hochberg" method or the "Holm" methods. All statistical analyses were performed with relevant packages in $\mathrm{R}$.

\section{Supplementary information}

Supplementary information accompanies this paper at https://doi.org/10. 1186/s12864-020-6703-0.

Additional file 1 : Figure S1. Flowchart of the study. Figure S2 Flowchart of the major steps involved in biomarker identification. Figure
S3. Plot of the Simpson and Pielou's evenness indices. Figure S4. Predictive accuracies of different random forest models. Figure S5. The variable importance of OTUs.

Additional file 2 : Table S1. Mapping file (metadata); Table S2: OTU table; Table S3: Taxonomy of OTUs; Table S4. a diversity indices. Table S5. Data for Venn diagram. Table S6. OTUs greater than $0.3 \%$ of the total reads. Table S7. $P$ values of genera (The results of compositional analysis using the ALDEx2 package at the genus level). Table S8. $P$ values of OTU (The results of compositional analysis using the ALDEx2 package at the OTU level). Table S9. OTUs greater than $0.001 \%$ of the total reads.

\section{Abbreviations}

ACPA: Anti-citrullinated protein antibody; ANA: Antinuclear antibody; AUC: Area under receiver operating characteristic curve; BMI: Body mass index; CD: Cluster of differentiation; CJADAS10: Clinical juvenile arthritis disease activity score 10; CRP: C-reactive protein; DCA: Decision curve analysis; DMARDs: Disease-modifying anti-rheumatic drugs; ESR: Erythrocyte sedimentation rate; Glo: Globulin; Hb: Hemoglobin; HC: Healthy children;

IFN: Interferon; IL: Interleukin; IQR: Interquartile range; JIA: Juvenile idiopathic arthritis; Neu: Neutrophil; OTUs: Operational taxonomic units; PCOA: Principal coordinate analysis; PI: Platelet; RF: Rheumatoid factor; ROC: Receiver operating characteristic curve; SCFAs: Short-chain fatty acids; TC: Total cholesterol; TNF: Tumor necrosis factor; WBC: While blood cell

\section{Acknowledgments}

The authors would like to thank Lawrence Kwok Leung Jung, MD, former chief of Division of Rheumatology, Children's National Medical Center in Washington D.C., for valuable advice about the study and critical review of the manuscript; the public representatives, Shudan Qian, and Lina Chen for their assistance in managing the project.

\section{Author's contributions}

X.Q. and M.L. designed the study and obtained funding. X.Y., W.Z., S.L., M.M., J.L., W.W.1 (Wenqin Wang), and W.W.2 were involved in patient recruitment. X.Y., M.M., J.L., and W.W.1 performed data collection. X.Q., M.L., Y.L., and X.Y. performed data analysis. X.Q. and Y.L. were involved in the selection of the analysis methods and the interpretation of the microbiome findings. X.Q., M.L., X.Z., and Y.L. drafted the manuscript. All authors were involved in the manuscript preparation and approved of the final version as written.

\section{Funding}

This research was funded by the Project of Young Talent in Medical Field in Zhejiang Province (2015-70) from the Health Commission of Zhejiang Province, and the Zhejiang Provincial Natural Science Foundation (LGF19H100002). The funding agencies had no role in the study design, data collection and analysis, decision to publish, or preparation of the manuscript.

\section{Availability of data and materials}

The 165 rDNA sequencing data are available in the NCBI SRA repository under BioProject PRJNA562467. The R codes and its input of the figures in the manuscript are available at https://github.com/qianxubo/microbiotastage-1.

\section{Ethics approval and consent to participate}

This study was approved by the Institutional Review Boards at 1) Children's Hospital, Zhejiang University School of Medicine (2018-IRB-031) and 2) Jinhua Municipal People's Hospital (MIK2018001). The principles outlined in the Declaration of Helsinki were followed. Informed consent was obtained before the participants were enrolled. For children 10 years of age or younger, their parents were asked to sign an informed consent form. For children older than 10 years of age, both the child and his/her parents were asked to sign an informed consent form.

Consent for publication

Not applicable.

Competing interests

The authors declare that they have no competing interests. 


\section{Author details}

'Department of Rheumatology Immunology and Allergy, Children's Hospital, Zhejiang University School of Medicine, Hangzhou, Zhejiang Province, China. ${ }^{2}$ State Key Laboratory of Plant Genomics, Institute of Genetics and Developmental Biology, Chinese Academy of Sciences, Beijing, China. ${ }^{3}$ Department of Scientific Research Management and Medical Education, Jinhua Hospital of Traditional Chinese Medicine, Jinhua, Zhejiang Province, China. ${ }^{4}$ Department of Paediatric Rheumatology, The Second Affiliated Hospital and Yuying Children's Hospital of Wenzhou Medical University, Wenzhou, Zhejiang Province, China. ${ }^{5}$ Nursing Department, Jiangnan Community Healthcare Center, Jinhua, Zhejiang Province, China. ${ }^{6}$ Department of Pediatrics, Wenling Maternal and Child Healthcare Hospital, Wenling, Zhejiang Province, China. ${ }^{7}$ Department of Pediatrics, Shaoxing People's Hospital, Shaoxing Hospital, Zhejiang University School of Medicine, Shaoxing, Zhejiang Province, China. ${ }^{8}$ Department of Rheumatology Immunology, Jinhua Municipal People's Hospital, Jinhua, Zhejiang Province, China. ${ }^{9}$ Department of Genetics, Institute of Genetics, Institute of Cell Biology, Zhejiang University School of Medicine, Hangzhou, Zhejiang Province, China.

Received: 23 January 2020 Accepted: 25 March 2020

Published online: 07 April 2020

\section{References}

1. Wu EY, Bryan AR, Rabinovich CE. Juvenile idiopathic arthritis. In: Kliegman RM, Stanton BF, St Geme III JW, Schor NF, editors. Nelson textbook of pediatrics. 20th ed. New York: Elsevier; 2015. p. 1160-70.

2. Prakken B, Albani S, Martini A. Juvenile idiopathic arthritis. Lancet. 2011; 377(9783):2138-49. https://doi.org/10.1016/S0140-6736(11)60244-4.

3. Shiff NJ, Oen K, Kroeker K, Lix LM. Trends in population-based incidence and prevalence of juvenile idiopathic arthritis in Manitoba, Canada. Arthritis Care Res. 2019;71(3):413-8. https://doi.org/10.1002/acr.23606.

4. Kip MMA, Currie G, Marshall DA, Grazziotin Lago L, Twilt M, Vastert SJ, Swart JF, Wulffraat N, Yeung RSM, Benseler SM, et al. Seeking the state of the art in standardized measurement of health care resource use and costs in juvenile idiopathic arthritis: a scoping review. Pediatr Rheumatol Online J. 2019;17(1):20. https://doi.org/10.1186/s12969-019-0321-x.

5. Shenoi S, Horneff G, Cidon M, Ramanan AV, Kimura Y, Quartier P, Foeldvari I, Zeft A, Lomax KG, Gregson J, et al. The burden of systemic juvenile idiopathic arthritis for patients and caregivers: an international survey and retrospective chart review. Clin Exp Rheumatol. 2018;36(5):920-8.

6. Angelis A, Kanavos P, Lopez-Bastida J, Linertova R, Serrano-Aguilar P, Network B-RR. Socioeconomic costs and health-related quality of life in juvenile idiopathic arthritis: a cost-of-illness study in the United Kingdom. BMC Musculoskelet Disord. 2016;17:321. https://doi.org/10.1186/s12891-0161129-1.

7. Charuvanij S, Chaiyadech C. Health-related quality of life in children with early-stage juvenile idiopathic arthritis. Musculoskeletal Care. 2019;17(2):21520. https://doi.org/10.1002/msc.1393.

8. Schlenner S, Pasciuto E, Lagou V, Burton O, Prezzemolo T, Junius S, Roca CP, Seillet C, Louis C, Dooley J, et al. NFIL3 mutations alter immune homeostasis and sensitise for arthritis pathology. Ann Rheum Dis. 2019;78(3):342-9. https://doi.org/10.1136/annrheumdis-2018-213764.

9. Nigrovic PA, Martinez-Bonet M, Thompson SD. Implications of juvenile idiopathic arthritis genetic risk variants for disease pathogenesis and classification. Curr Opin Rheumatol. 2019. https://doi.org/10.1097/BOR. 0000000000000637.

10. Franca CMP, Sallum AME, Braga ALF, Strufaldi FL, Silva CAA, Farhat SCL. Risk factors associated with juvenile idiopathic arthritis: exposure to cigarette smoke and air pollution from pregnancy to disease diagnosis. J Rheumatol. 2018;45(2):248-56. https://doi.org/10.3899/jrheum.161500.

11. Hersh AO, Prahalad S. Genetics of juvenile idiopathic arthritis. Rheum Dis Clin N Am. 2017;43(3):435-48. https://doi.org/10.1016/.j.rdc.2017.04.007.

12. Smolen JS, Aletaha D, Barton A, Burmester GR, Emery P, Firestein GS, Kavanaugh A, Mclnnes IB, Solomon DH, Strand V, et al. Rheumatoid arthritis. Nat Rev Dis Primers. 2018;4:18001. https://doi.org/10.1038/nrdp.2018.1.

13. Sparks JA, Costenbader KH. Rheumatoid arthritis in 2017: protective dietary and hormonal factors brought to light. Nat Rev Rheumatol. 2018;14(2):71-2. https://doi.org/10.1038/nrrheum.2017.216.
14. Van de Wiele T, Van Praet JT, Marzorati M, Drennan MB, Elewaut D. How the microbiota shapes rheumatic diseases. Nat Rev Rheumatol. 2016;12(7):398411. https://doi.org/10.1038/nrrheum.2016.85.

15. Onuora S. Juvenile idiopathic arthritis: evidence of gut-driven inflammation in new JIA. Nat Rev Rheumatol. 2017;13(11):632. https://doi.org/10.1038/ nrrheum.2017.168.

16. De Filippo C, Di Paola M, Giani T, Tirelli F, Cimaz R. Gut microbiota in children and altered profiles in juvenile idiopathic arthritis. J Autoimmun. 2019;98:1-12. https://doi.org/10.1016/j.jaut.2019.01.001.

17. Di Paola M, Cavalieri D, Albanese D, Sordo M, Pindo M, Donati C, Pagnini I, Giani T, Simonini G, Paladini A, et al. Alteration of fecal microbiota profiles in juvenile idiopathic arthritis. Associations with HLA-B27 allele and disease status. Front Microbiol. 2016;7:1703. https://doi.org/10.3389/fmicb.2016. 01703.

18. Tejesvi MV, Arvonen M, Kangas SM, Keskitalo PL, Pirttila AM, Karttunen TJ, Vahasalo P. Faecal microbiome in new-onset juvenile idiopathic arthritis. Eur J Clin Microbiol Infect Dis. 2016;35(3):363-70. https://doi.org/10.1007/ s10096-015-2548-X.

19. Aggarwal A, Sarangi AN, Gaur P, Shukla A, Aggarwal R. Gut microbiome in children with enthesitis-related arthritis in a developing country and the effect of probiotic administration. Clin Exp Immunol. 2017;187(3):480-9. https://doi.org/10.1111/cei.12900.

20. van Dijkhuizen EHP, Del Chierico F, Malattia C, Russo A, Pires Marafon D, Ter Haar NM, Magni-Manzoni S, Vastert SJ, Dallapiccola B, Prakken B, et al. Microbiome analytics of the gut microbiota in patients with juvenile idiopathic arthritis: a longitudinal observational cohort study. Arthritis Rheumatol. 2019;71(6):1000-10. https://doi.org/10.1002/art.40827.

21. Zhang X, Zhang D, Jia H, Feng Q, Wang D, Liang D, Wu X, Li J, Tang L, Li Y, et al. The oral and gut microbiomes are perturbed in rheumatoid arthritis and partly normalized after treatment. Nat Med. 2015;21(8):895-905. https:// doi.org/10.1038/nm.3914.

22. Sanna S, van Zuydam NR, Mahajan A, Kurilshikov A, Vich Vila A, Vosa U, Mujagic Z, Masclee AAM, Jonkers D, Oosting M, et al. Causal relationships among the gut microbiome, short-chain fatty acids and metabolic diseases. Nat Genet. 2019;51(4):600-5. https://doi.org/10.1038/s41588-019-0350-x.

23. Pianta A, Arvikar S, Strle K, Drouin EE, Wang Q, Costello CE, Steere AC. Evidence of the immune relevance of Prevotella copri, a gut microbe, in patients with rheumatoid arthritis. Arthritis Rheumatol. 2017;69(5):964-75. https://doi.org/10.1002/art.40003.

24. Manfredo Vieira S, Hiltensperger M, Kumar V, Zegarra-Ruiz D, Dehner $C$, Khan N, Costa FRC, Tiniakou E, Greiling T, Ruff W, et al. Translocation of a gut pathobiont drives autoimmunity in mice and humans. Science. 2018; 359(6380):1156-61. https://doi.org/10.1126/science.aar7201.

25. Odamaki T, Kato K, Sugahara H, Hashikura N, Takahashi S, Xiao JZ, Abe F, Osawa R. Age-related changes in gut microbiota composition from newborn to centenarian: a cross-sectional study. BMC Microbiol. 2016;16:90. https://doi.org/10.1186/s12866-016-0708-5.

26. Rizzetto L, Fava F, Tuohy KM, Selmi C. Connecting the immune system, systemic chronic inflammation and the gut microbiome: the role of sex. J Autoimmun. 2018;92:12-34. https://doi.org/10.1016/j.jaut.2018.05.008.

27. Sun L, Ma L, Ma Y, Zhang F, Zhao C, Nie Y. Insights into the role of gut microbiota in obesity: pathogenesis, mechanisms, and therapeutic perspectives. Protein Cell. 2018;9(5):397-403. https://doi.org/10.1007/s13238018-0546-3.

28. Bui TP, de Vos WM, Plugge CM. Anaerostipes rhamnosivorans sp. nov., a human intestinal, butyrate-forming bacterium. Int J Syst Evol Microbiol. 2014;64(Pt 3):787-93. https://doi.org/10.1099/ijs.0.055061-0.

29. Schwiertz A, Hold GL, Duncan SH, Gruhl B, Collins MD, Lawson PA, Flint HJ, Blaut M. Anaerostipes caccae gen. Nov., sp. nov., a new saccharolytic, acetate-utilising, butyrate-producing bacterium from human faeces. Syst Appl Microbiol. 2002;25(1):46-51. https://doi.org/10.1078/0723-2020-00096.

30. Meehan CJ, Beiko RG. A phylogenomic view of ecological specialization in the Lachnospiraceae, a family of digestive tract-associated bacteria. Genome Biol Evol. 2014;6(3):703-13. https://doi.org/10.1093/gbe/evu050.

31. Tamanai-Shacoori Z, Smida I, Bousarghin L, Loreal O, Meuric V, Fong SB, Bonnaure-Mallet M, Jolivet-Gougeon A. Roseburia spp.: a marker of health? Future Microbiol. 2017;12:157-70. https://doi.org/10.2217/fmb-2016-0130.

32. Integrative HMP (iHMP) Research network Consortium. The Integrative human microbiome project. Nature. 2019;569(7758):641-8. https://doi.org/ 10.1038/s41586-019-1238-8. 
33. Cao H, Xia T, Li Y, Xu Z, Bougouffa S, Lo YK, Bajic VB, Luo H, Woo PCY, Yan A. Uncoupled quorum sensing modulates the interplay of virulence and resistance in a multidrug-resistant clinical Pseudomonas aeruginosa isolate belonging to the MLST550 clonal complex. Antimicrob Agents Chemother. 2019;63(4):e01944-18. https://doi.org/10.1128/AAC.01944-18.

34. Cao H, Lai Y, Bougouffa S, Xu Z, Yan A. Comparative genome and transcriptome analysis reveals distinctive surface characteristics and unique physiological potentials of Pseudomonas aeruginosa ATCC 27853. BMC Genomics. 2017;18(1):459. https://doi.org/10.1186/s12864-017-3842-z.

35. Alves M, Pereira A, Vicente C, Matos P, Henriques J, Lopes H, Nascimento F, Mota M, Correia A, Henriques I. The role of bacteria in pine wilt disease: insights from microbiome analysis. FEMS Microbiol Ecol. 2018;94(7):fiy077. https://doi.org/10.1093/femsec/fiy077.

36. Bolyen E, Rideout JR, Dillon MR, Bokulich NA, Abnet CC, Al-Ghalith GA, Alexander H, Alm EJ, Arumugam M, Asnicar F, et al. Reproducible, interactive, scalable and extensible microbiome data science using QIIME 2. Nat Biotechnol. 2019;37(8):852-7. https://doi.org/10.1038/s41587-019-0209-9.

37. Liu YX, Qin Y, Bai Y. Reductionist synthetic community approaches in root microbiome research. Curr Opin Microbiol. 2019;49:97-102. https://doi.org/ 10.1016/j.mib.2019.10.010.

38. Colldahl $\mathrm{H}$. The intestinal flora in patients with bronchial asthma and rheumatoid arthritis. Acta Allergol. 1965;20:94-104.

39. Arvonen M, Stoll ML. Juvenile idiopathic arthritis. In: Ragab G, Atkinson TP, Stoll ML, editors. The microbiome in rheumatic diseases and infection. 1st ed. Switzerland: Springer International Publishing; 2018. p. 221-37. https:// doi.org/10.1007/978-3-319-79026-8.

40. Yatsunenko T, Rey FE, Manary MJ, Trehan I, Dominguez-Bello MG, Contreras M, Magris M, Hidalgo G, Baldassano RN, Anokhin AP, et al. Human gut microbiome viewed across age and geography. Nature. 2012;486(7402):2227. https://doi.org/10.1038/nature11053.

41. Gaulke CA, Sharpton TJ. The influence of ethnicity and geography on human gut microbiome composition. Nat Med. 2018;24(10):1495-6. https:// doi.org/10.1038/s41591-018-0210-8.

42. Gilbert JA, Blaser MJ, Caporaso JG, Jansson JK, Lynch SV, Knight R. Current understanding of the human microbiome. Nat Med. 2018;24(4):392-400. https://doi.org/10.1038/nm.4517.

43. Lee JJY, Schneider R. Systemic juvenile idiopathic arthritis. Pediatr Clin N Am. 2018;65(4):691-709. https://doi.org/10.1016/j.pcl.2018.04.005.

44. Ombrello MJ, Arthur VL, Remmers EF, Hinks A, Tachmazidou I, Grom AA, Foell D, Martini A, Gattorno M, Ozen S, et al. Genetic architecture distinguishes systemic juvenile idiopathic arthritis from other forms of juvenile idiopathic arthritis: clinical and therapeutic implications. Ann Rheum Dis. 2017;76(5):906-13. https://doi.org/10.1136/annrheumdis-2016210324.

45. Xia Y, Sun J, Chen D. Community diversity measures and calculations. In: Xia Y, Sun J, Chen D, editors. Statistical analysis of microbiome data with R. 1st ed. Singapore: Springer Singapore; 2018. p. 167-90. https://doi.org/10.1007/ 978-981-13-1534-3.

46. Breban M, Tap J, Leboime A, Said-Nahal R, Langella P, Chiocchia G, Furet JP, Sokol $\mathrm{H}$. Faecal microbiota study reveals specific dysbiosis in spondyloarthritis. Ann Rheum Dis. 2017;76(9):1614-22. https://doi.org/10. 1136/annrheumdis-2016-211064.

47. Stoll ML, Weiss PF, Weiss JE, Nigrovic PA, Edelheit BS, Bridges SL Jr, Danila MI, Spencer CH, Punaro MG, Schikler K, et al. Age and fecal microbial strainspecific differences in patients with spondyloarthritis. Arthritis Res Ther. 2018;20(1):14. https://doi.org/10.1186/s13075-018-1510-6.

48. Morotomi M, Nagai F, Sakon H, Tanaka R. Dialister succinatiphilus sp. nov. and Barnesiella intestinihominis sp. nov., isolated from human faeces. Int J Syst Evol Microbiol. 2008;58(Pt 12):2716-20. https://doi.org/10.1099/ijs.0. 2008/000810-0.

49. Tito RY, Cypers H, Joossens M, Varkas G, Van Praet L, Glorieus E, Van den Bosch F, De Vos M, Raes J, Elewaut D. Brief report: dialister as a microbia marker of disease activity in Spondyloarthritis. Arthritis Rheumatol. 2017; 69(1):114-21. https://doi.org/10.1002/art.39802.

50. Stewart CJ, Ajami NJ, O'Brien JL, Hutchinson DS, Smith DP, Wong MC, Ross MC, Lloyd RE, Doddapaneni H, Metcalf GA, et al. Temporal development of the gut microbiome in early childhood from the TEDDY study. Nature. 2018; 562(7728):583-8. https://doi.org/10.1038/s41586-018-0617-x.

51. Kundu P, Blacher E, Elinav E, Pettersson S. Our gut microbiome: the evolving inner self. Cell. 2017;171(7):1481-93. https://doi.org/10.1016/j.cell.2017.11.024.
52. David LA, Maurice CF, Carmody RN, Gootenberg DB, Button JE, Wolfe BE, Ling AV, Devlin AS, Varma Y, Fischbach MA, et al. Diet rapidly and reproducibly alters the human gut microbiome. Nature. 2014;505(7484):55963. https://doi.org/10.1038/nature12820.

53. Furusawa Y, Obata Y, Fukuda S, Endo TA, Nakato G, Takahashi D, Nakanishi Y, Uetake C, Kato K, Kato T, et al. Commensal microbe-derived butyrate induces the differentiation of colonic regulatory T cells. Nature. 2013; 504(7480):446-50. https://doi.org/10.1038/nature12721.

54. Hui W, Yu D, Cao Z, Zhao X. Butyrate inhibit collagen-induced arthritis via Treg/IL-10/Th17 axis. Int Immunopharmacol. 2019;68:226-33. https://doi.org/ 10.1016/j.intimp.2019.01.018

55. Luu M, Pautz S, Kohl V, Singh R, Romero R, Lucas S, Hofmann J, Raifer H, Vachharajani N, Carrascosa LC, et al. The short-chain fatty acid pentanoate suppresses autoimmunity by modulating the metabolic-epigenetic crosstalk in lymphocytes. Nat Commun. 2019;10(1):760. https://doi.org/10.1038/ s41467-019-08711-2.

56. Zhao L, Zhang F, Ding X, Wu G, Lam YY, Wang X, Fu H, Xue X, Lu C, Ma J, et al. Gut bacteria selectively promoted by dietary fibers alleviate type 2 diabetes. Science. 2018:359(6380):1151-6. https://doi.org/10.1126/science. aao5774.

57. Steyerberg EW. Evaluation of clinical usefulness. In: Clinical prediction models. 2nd ed. New York City: Springer International Publishing; 2019. p. 309-28. https://doi.org/10.1007/978-3-030-16399-0.

58. Van Calster B, Wynants L, Verbeek JFM, Verbakel JY, Christodoulou E, Vickers AJ, Roobol MJ, Steyerberg EW. Reporting and interpreting decision curve analysis: a guide for investigators. Eur Urol. 2018;74(6):796-804. https://doi. org/10.1016/j.eururo.2018.08.038.

59. Vickers AJ, Elkin EB. Decision curve analysis: a novel method for evaluating prediction models. Med Decis Mak. 2006;26(6):565-74. https://doi.org/10. 1177/0272989X06295361.

60. Zhang Z, Rousson V, Lee WC, Ferdynus C, Chen M, Qian X, Guo Y. Written on behalf of AMEB-DCTCG: decision curve analysis: a technical note. Ann Transl Med. 2018;6(15):308. https://doi.org/10.21037/atm.2018.07.02.

61. Petty RE, Southwood TR, Manners P, Baum J, Glass DN, Goldenberg J, He X, Maldonado-Cocco J, Orozco-Alcala J, Prieur AM, et al. International league of associations for rheumatology classification of juvenile idiopathic arthritis: second revision, Edmonton, 2001. J Rheumatol. 2004;31(2):390-2.

62. Ashworth A. Nutrition, food security, and health. In: Kliegman RM, Stanton BF, St Geme III JW, Schor NF, editors. Nelson textbook of pediatrics. 20th ed. New York: Elsevier; 2015. p. 295-306.

63. Gahagan S. Overweight and obesity. In: Kliegman RM, Stanton BF, St Geme III JW, Schor NF, editors. Nelson textbook of pediatrics. 20th ed. New York: Elsevier; 2015. p. 307-16.

64. Caporaso JG, Kuczynski J, Stombaugh J, Bittinger K, Bushman FD, Costello EK, Fierer N, Pena AG, Goodrich JK, Gordon Jl, et al. QIIME allows analysis of high-throughput community sequencing data. Nat Methods. 2010;7(5):3356. https://doi.org/10.1038/nmeth.f.303.

65. Douglas G, Comeau A, Langille MI. Processing a $16 \mathrm{~S}$ rRNA sequencing dataset with the microbiome helper workflow. In: Beiko R, Hsiao W, Parkinson J, editors. Microbiome analysis. 1st ed. Totowa: Humana Press; 2018. p. 131-41. https://doi.org/10.1007/978-1-4939-8728-3.

66. McDonald D, Price MN, Goodrich J, Nawrocki EP, DeSantis TZ, Probst A, Andersen GL, Knight R, Hugenholtz P. An improved Greengenes taxonomy with explicit ranks for ecological and evolutionary analyses of bacteria and archaea. ISME J. 2012;6(3):610-8. https://doi.org/10.1038/ismej.2011.139.

67. Bokulich NA, Subramanian S, Faith JJ, Gevers D, Gordon JI, Knight R, Mills DA, Caporaso JG. Quality-filtering vastly improves diversity estimates from Illumina amplicon sequencing. Nat Methods. 2013;10(1):57-9. https://doi. org/10.1038/nmeth.2276.

68. vegan: community ecology package [https://cran.r-project.org/web/ packages/vegan/index.html]

69. McMurdie PJ, Holmes S. phyloseq: an R package for reproducible interactive analysis and graphics of microbiome census data. PLoS One. 2013;8(4): e61217. https://doi.org/10.1371/journal.pone.0061217.

70. Xia Y, Sun J, Chen D. Compositional analysis of microbiome data. In: Xia Y, Sun J, Chen D, editors. Statistical analysis of microbiome data with R. 1st ed. Singapore: Springer Singapore; 2018. p. 331-93. https://doi.org/10.1007/978981-13-1534-3.

71. Borcard D, Gillet F, Legendre P. Unconstrained ordination. In: Borcard D, Gillet F, Legendre P, editors. Numerical ecology with R. 2nd ed. Switzerland: 
Springer International Publishing; 2018. p. 151-201. https://doi.org/10.1007/ 978-3-319-71404-2.

72. Yu G, Lam TT, Zhu H, Guan Y. Two methods for mapping and visualizing associated data on phylogeny using Ggtree. Mol Biol Evol. 2018;35(12): 3041-3. https://doi.org/10.1093/molbev/msy194.

73. Analysis of differential abundance taking sample variation into account (ALDEx2) [https://bioconductor.org/packages/release/bioc/html/ALDEx2. html].

74. Wickham H. ggplot2: elegant graphics for data analysis. Springer-Verlag New York., 2 edn. New York: Springer International Publishing; 2016.

75. psych: procedures for personality and psychological research [https://CRAN. R-project.org/package=psych].

76. corrplot: visualization of a correlation matrix [https://cran.r-project.org/web/ packages/corrplot/index.html].

77. Breiman L. Random forests. Mach Learn. 2001:45(1):5-32.

78. randomForest: Breiman and Cutler's random forests for classification and regression [https://cran.r-project.org/web/packages/randomForest/index. $h \mathrm{tml}]$.

79. Sing $T$, Sander $\mathrm{O}$, Beerenwinkel $\mathrm{N}$, Lengauer T. ROCR: visualizing classifier performance in R. Bioinformatics. 2005;21 (20):3940-1. https://doi.org/10. 1093/bioinformatics/bti623.

80. Zhang Z. Decision tree modeling using R. Ann Transl Med. 2016:4(15):275. https://doi.org/10.21037/atm.2016.05.14.

81. Xia Y, Sun J, Chen D. Power and sample size calculations for microbiome data. In: Xia Y, Sun J, Chen D, editors. Statistical analysis of microbiome data with R. 1st ed. Singapore: Springer Singapore; 2018. p. 129-66. https://doi. org/10.1007/978-981-13-1534-3.

\section{Publisher's Note}

Springer Nature remains neutral with regard to jurisdictional claims in published maps and institutional affiliations.

Ready to submit your research? Choose BMC and benefit from:

- fast, convenient online submission

- thorough peer review by experienced researchers in your field

- rapid publication on acceptance

- support for research data, including large and complex data types

- gold Open Access which fosters wider collaboration and increased citations

- maximum visibility for your research: over $100 \mathrm{M}$ website views per year

At $\mathrm{BMC}$, research is always in progress.

Learn more biomedcentral.com/submissions 